\title{
Controlled lateral growth of silica nanowires
}

\author{
Tae-Hyun Kim, Avi Shalav, and Robert G. Elliman* \\ Department of Electronic Materials Engineering, Research School of Physics and Engineering, The Austra- \\ lian National University, Canberra, ACT 0200, Australia \\ ${ }^{*}$ Corresponding author: Email rob.elliman@anu.edu.au)
}

\begin{abstract}
This study investigates the controlled lateral growth of amorphous silica nanowires using the Si active oxidation as the dominant $\mathrm{SiO}$ vapour precursor for nanowire growth. The development of complex multi-layered core shell nanowire structures using a combination of both active Si oxidation and secondary coating techniques is also demonstrated.
\end{abstract}

1 Introduction: Control over nanowire growth is an important requirement for future nanotechnology devices since the physical structure and chemical properties of nanowires are critically affected by diameter, length, atomic composition and nanowire density [1-2]. Various methods based on the Vapour-LiquidSolid (VLS) mechanism have been introduced and developed to control the diameter of nanowires.

Amorphous silica nanowires have great potential for biological and environmental sensing and optical devices due to their large surface-to-volume fraction, chemical inertness, and optical transparency. Also, silica nanowires are suitable to use a 'core' or 'shell' structure with doping or coating materials for functionalized applications [3-4].

In this study, we report the lateral growth of silica nanowires grown via the active oxidation of the underlying Si substrate. Secondary coatings can be readily added to these silica core structures via sputtering and vapour phase deposition processes. Upon annealing at higher temperatures, $\mathrm{SiO}$ is adsorbed onto the walls of the coated nanowires resulting in the development of more complex multilayered core-shell nanowires.

2 Experimental details: A metal (Au) catalyst layer with the thickness of $10 \mathrm{~nm}$ on (100) silicon wafer was deposited by sputter depostiona and subse- quently annealed in a quartz tube furnace at temperature $1100^{\circ} \mathrm{C}$ for various times between 5 minutes and 2 hours in $\mathrm{N}_{2}$ gas ambient. Under these conditions, the $\mathrm{Si}$ substrate undergoes active oxidation, releasing volatile $\mathrm{SiO}$ vapour. This is the primary vapour source utilized during the growth of silica nanowires via the VLS mechanism. The as-grown silica nanowires were the coated by Atomic Layer Deposition (ALD) or by sputtering with secondary materials including $\mathrm{HfO}_{2}$ and Erbium and also re-annealed at a temperature of $1100^{\circ} \mathrm{C}$ in $\mathrm{N}_{2}$ ambient. At this temperature, $\mathrm{SiO}$ is again created and coats the already coated nanowires.

The structures and morphology of the samples were studied by scanning electron microscopy (SEM) and highresolution transmission electron microscopy (HRTEM).

\section{Overview of Results:}

Fig. 1 shows SEM images of nanowires after an annealing time of a) 20 , b) 40 , c) 60 , and d) $120 \mathrm{~min}$. showing lateral growth of the nanowires with time.

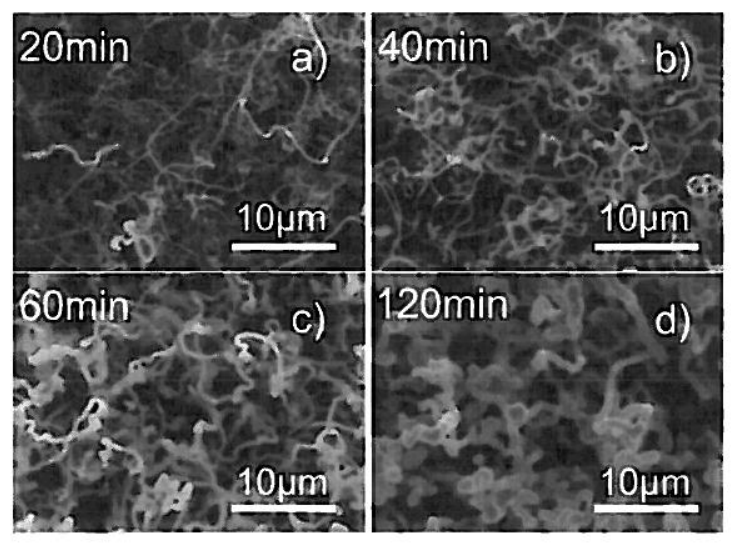

Fig. 1: SEM images of $10 \mathrm{~nm}$ Au on Si after annealing at $1100^{\circ} \mathrm{C}$ for a) $20 \mathrm{~min}$, b) $40 \mathrm{~min}$, c) 1 hour, and d) 2 hours. 
As shown in Fig. 2 a), the diameter of the nanowires increases linearly with increasing annealing time (note: error bars were determined based on the standard deviation from mean diameter of nanowires). The nanowires are undergo lateral growth involving the adsorption of $\mathrm{SiO}$ vapour onto the already grown nanowire surfaces.
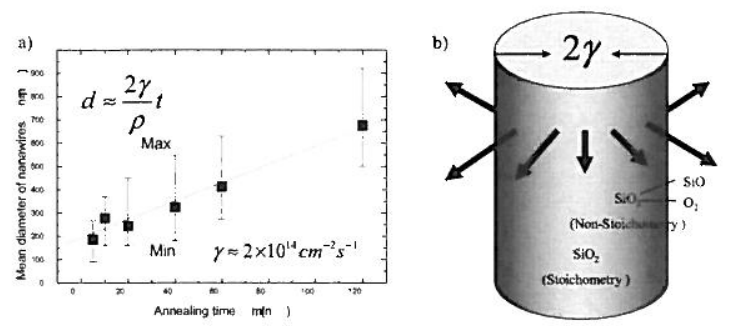

Fig. 2: a) Diameter of nanowires as a function of annealing time and b) schematic illustration of lateral growth.

The lateral growth can explained using a simple relationship between incident $\mathrm{SiO}$ flux (assumed constant) and the increase in nanowire volumetric size. For a cylindrical nanowire exposed to a fixed concentration of vapour, the rate of change of volume can be written as: $d V / d t=\gamma \mathrm{A} / \rho$, where $\gamma$ is the atomic (or molecular) flux deposited onto the cylinder surface (in $\left.\mathrm{cm}^{-2} \cdot \mathrm{s}^{-1}\right), A=2 \pi \mathrm{rl}$ is the approximate surface area of the cylinder, with $r$ the radius and $I$ the length of the cylinder, and $\rho$ is the atomic (molecular) density of the deposited film (atoms. $\mathrm{cm}^{-3}$ ). Since the volume of the cylinder is $V=\pi r^{2}$, the change in the nanowire radius is: $r=2 \gamma \mathrm{t} / \rho$, that is, a linear increase with time, as observed. A linear fit to the data in Fig. 2 yields a value for $\gamma$ of around $2 \times 10^{14} \mathrm{~cm}^{-2} \mathrm{~s}^{-1}$ or around 0.1 monolayer per second.
By using lateral growth and secondary coating processes, multi core-shell structures were fabricated, as shown in Fig. 3. $\mathrm{HfO}_{2}$ or $\mathrm{Er}$ on as-grown silica nanowire (core) were coated by ALD or sputter depostion and re-annealed in furnace. After re-annealing, coating process was repeated. The surface of $\mathrm{HfO}_{2}$ or $\mathrm{Er}$ coated nanowires were coated by a silicabased layer formed by the adsorption of $\mathrm{SiO}$ vapour.

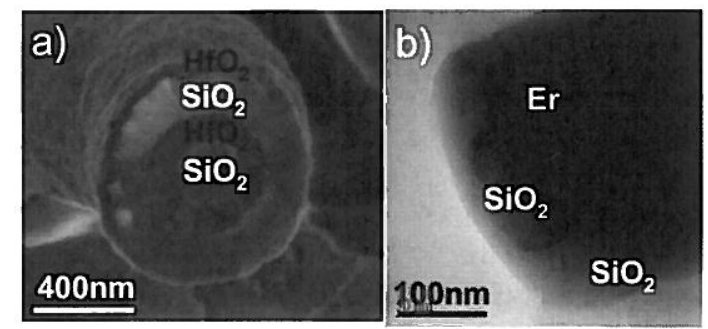

Fig. 3: a) $\mathrm{SiO}_{2} / \mathrm{HfO}_{2} / \mathrm{SiO}_{2} / \mathrm{HfO}_{2}$ coaxial and b) $\mathrm{SiO}_{2} / \mathrm{Er} / \mathrm{SiO}_{2}$ coaxial nanowires grown using lateral growth

\section{Acknowledgements}

The authors acknowledge Mr David Lleyellyn for assistance with TEM sample preparation, and the Australian Research Council for partial financial support through the ARC Linkage Project scheme.

\section{References}

[1] Y. Cui, X. F. Duan, J. T. Hu, C. M. Lieber, "Doping and electrical transport in silicon nanowires", J. Phy. Che. B 2000, 104, 5213

[2] R. G. Elliman, A. R. Wilkinson, T. H. Kim, P. K. Sekhar, S. Bhansali, "Optical emission from erbium-doped silica nanowires", J. Appl. Phy 2008, 103.

[3] A. Shalav, T. H. Kim, and R. G. Elliman "Secondary growth and photoluminescence form erbirm implanted silica nanowires", J. Appl. Phys. 2010, 107 (4), 046101.

[4] P. K. Sekhar, A. R. Wilkinson, R. G. Elliman T. H. Kim and S. Bhansali, "Erbium emission from nanoengineered silicon surface", J. Phys. Chem. C, 2008, 112, 20109. 